\title{
Halo Orbit Mission Correction Maneuvers Using Optimal Control
}

\author{
Radu Serban, Wang S. Koon ${ }^{\dagger}$ Martin W. Lo $\ddagger$ Jerrold E. Marsden; \\ Linda R. Petzold, Shane D. Ross, and Roby S. Wilson ${ }^{\ddagger}$
}

March 7, 2000

\begin{abstract}
This paper addresses the computation of the required trajectory correction maneuvers (TCM) for a halo orbit space mission to compensate for the launch velocity errors introduced by inaccuracies of the launch vehicle. By combining dynamical systems theory with optimal control techniques, we are able to provide a compelling portrait of the complex landscape of the trajectory design space. This approach enables automation of the analysis to perform parametric studies that simply were not available to mission designers a few years ago, such as how the magnitude of the errors and the timing of the first trajectory correction maneuver affects the correction $\Delta V$. The impetus for combining dynamical systems theory and optimal control in this problem arises from design issues for the Genesis Discovery mission being developed for NASA by the Jet Propulsion Laboratory.
\end{abstract}

\section{Introduction and Background}

\subsection{The Genesis Mission}

Genesis is a solar wind sample return mission (see Lo et al., [1998]). It is one of NASA's first robotic sample return missions and is scheduled for launch in January 2001 to a halo orbit in the vicinity of the Sun-Earth $L_{1}$ Lagrange point; $L_{1}$ is one of the five equilibrium points in the circular restricted three body problem (CRTBP). See Figure 1 for a depiction of the Genesis trajectory.

In the standard convention, $L_{1}$ is the unstable equilibrium point between the Sun and the Earth at roughly 1.5 million $\mathrm{km}$ from the Earth in the direction of the Sun. Once there, the spacecraft will remain in the halo orbit for two years to collect solar wind samples before returning them to the Earth for study into the origins

*Department of Mechanical and Environmental Engineering, University of California, Santa Barbara, CA 93106. This research was supported in part by the NSFKDI grant number NSF ATM-9873133.

${ }^{\dagger}$ Control and Dynamical Systems, Caltech 107-81, Pasadena, CA 91125.

${ }^{t}$ Navigation and Flight Mechanics, Jet Propulsion Laboratory M/S: 301-140L, 4800 Oak Grove Drive, Pasadena, CA 91109. 

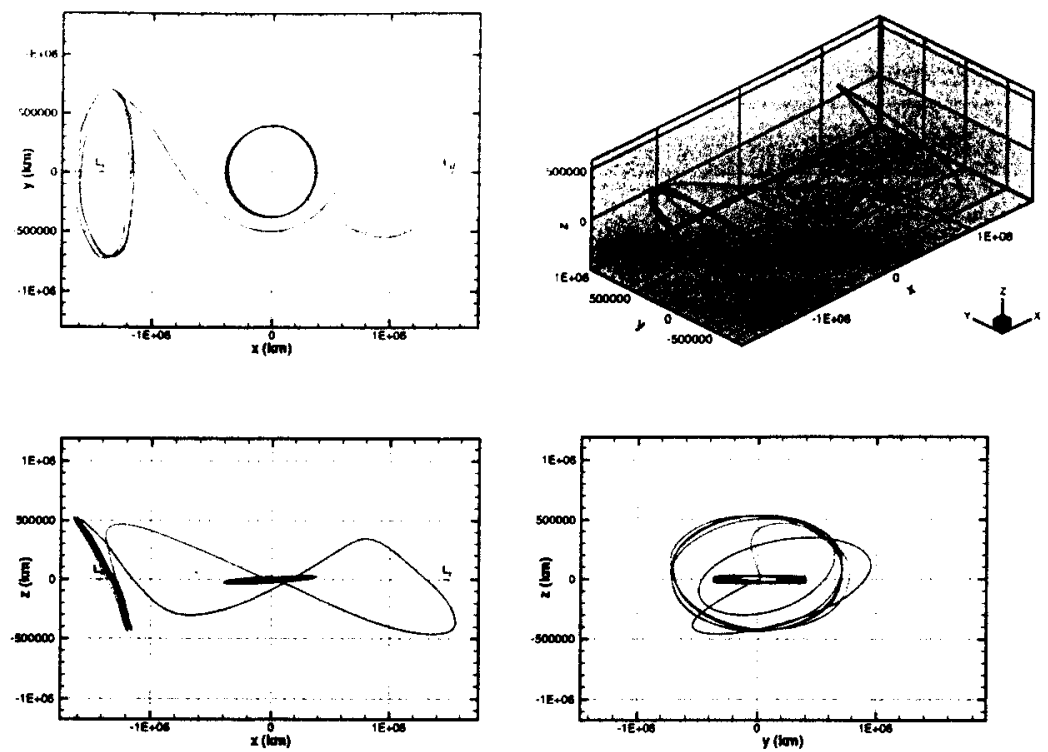

Figure 1: Genesis Trajectory, XY, XZ, and YZ Projections.

of the solar system. Figure 1 shows three orthographic projections of the Genesis trajectory: the transfer to the halo, halo orbit itself, and the return to Earth. These figures are plotted in a rotating frame, which is often used in the study of the threebody problem. The frame is defined by fixing the $\mathrm{X}$-axis along the Sun-Earth line, the $\mathrm{Z}$-axis in the direction normal to the ecliptic, and the $\mathrm{Y}$-axis completing a righthanded coordinate system. Viewed from the Earth in the YZ-projection, the orbit appears as a halo around the Sun, hence its name (originally named for lunar halo orbits by Farquhar).

The Genesis trajectory is the first mission to be fully designed using dynamical systems theory (see Howell, Barden and Lo [1997]). Notice in Figure 1 that the trajectory travels between neighborhoods of the $L_{1}$ and $L_{2}$ libration points with the purpose of returning the samples to Earth $\left(L_{2}\right.$ is roughly 1.5 million $\mathrm{km}$ on the opposite side of the Earth from the Sun). In dynamical systems theory, this is called a heteroclinic connection between the $L_{1}$ and $L_{2}$ regions. One of the attractive features of this design is the fact that the three year mission, from launch all the way back to Earth return, requires only a single small deterministic maneuver (less than $6 \mathrm{~m} / \mathrm{s}$ ) when injecting onto the halo orbit! It is extremely difficult to use traditional classical algorithms to find such a near-optimal solution, so the design of such a low energy trajectory is facilitated by using dynamical systems methods. This is achieved by using the stable and unstable manifolds as guides in determining the end-to-end trajectory. 


\subsection{Halo Orbits}

Halo orbits are large three dimensional orbits shaped like the exges of a potato chip. The Y-amplitude of the Genesis halo orbit, which extends from the X-axis to the maximum $\mathrm{Y}$-value of the orbit, is about $780,000 \mathrm{~km}$. Note that this is bigger than the radius of the orbit of the Moon, which is about $380,000 \mathrm{~km}$. The computation of halo orbits follows standard nonlinear trajectory computation algorithms based on parallel shooting. Due to the sensitivity of the problem, an accurate first guess is essential, since the halo orbit is actually an unstable orbit (albeit with a fairly long time constant in the Sun-Earth system). This first guess is provided by a high order analytic expansion of minimum 3rd order using the Lindstedt-Poincaré method. For details see Llibre, Martinez and Simó [1985], Howell and Pernicka [1988], and Parker and Chua [1989].

In the CRTBP model, halo orbits are both periodic and time independent. However, if we take into account all the gravitational and non-gravitational effects of the full solar system (including those not modeled in the CRTBP), halo orbits are in fact quasiperiodic and time dependent. Like the $L_{1}$ equilibrium point, which is the generator of these families of unstable quasiperiodic orbits, the halo orbit is also an unstable orbit, behaving dynamically like a saddle point in the directions of spectrally unstable and stable eigenvalues. There is an entire family of asymptotic trajectories that departs from the halo orbit called the unstable manifold; there is also an entire family of asymptotic trajectories which wind onto the halo orbit called the stable manifold (see, for example, Wiggins [1990]). Each of these families form a two dimensional surface that is, roughly speaking, a twisted tubular surface emanating from the halo orbit.

Simó, Gómez, Llibre and Martinez [1987] were the first to study these invariant manifolds of the halo orbit and apply them to the design of the SOHO mission. SOHO did not require the delicate controls provided by this theory, so the actual mission was flown using the classical methods developed at NASA by Farquhar and Dunham (see, for example, Farquhar, Muhonen, Newman and Heuberger [1980]). For Genesis, however, these manifolds are absolutely crucial to return the samples to Earth and land at a specified site (a requirement not imposed on SOHO or previous libration point missions). The stable manifold, which winds onto the halo orbit, is used to design the transfer trajectory which delivers the Genesis spacecraft from launch to insertion onto the halo orbit (HOI). The unstable manifold, which winds off of the halo orbit, is used to design the return trajectory which brings the spacecraft and its precious samples back to Earth via the nearly heteroclinic connection. See Koon, Lo, Marsden and Ross [2000] for the current state of the computation of homoclinic and heteroclinic orbits in this problem.

\subsection{The Transfer to the Halo Orbit}

The transfer trajectory is designed using the following procedure. A halo orbit $H(t)$ is first selected, where $t$ represents time. The stable manifold of $H$, denoted $W^{s}$, consists of a family of asymptotic trajectories which take infinite time to wind onto $H$. These asymptotic solutions cannot be found numerically and are impractical for 
space missions where the transfor time needs to be just a few months. However, there is a family of trajectories that lie arbitrarily close to $W^{s}$ that require just a few months to transfer between Earth and the halo orbit. These trajectories are said to shadow the stable manifold. It is these shadow trajectories that we can compute and that are extremely useful to the design of the Genesis transfer trajectory.

A simple way to compute an approximation of $W^{s}$ is provided by Parker and Chua [1989] and is based on Floquet theory. The basic idea is to linearize the equations of motion about the periodic orbit and then use the monodromy matrix provided by Floquet theory to generate a linear approximation of the stable manifold associated with the halo orbit. The linear approximation, in the form of a state vector, is integrated in the nonlinear equations of motion to produce the approximation of the stable manifold. In the case of quasiperiodic orbits that are not too far from periodic orbits, one approximates the orbit as periodic and the same algorithm is applied to compute approximations of $W^{s}$ (see Howell, Barden and Lo [1997]; see also Gómez, Masdemont and Simó [1993]). For engineering purposes, at least for space missions, this seems to work well. Recently, a more refined approach based on reduction to the center manifold (or neutrally stable manifold) is provided by Jorba and Masdemont [1999].

In this paper, we will assume that the halo orbit, $H(t)$, and the stable manifold $M(t)$ are fixed and provided. Hence we will not dwell further on the theory of their computation which is well covered in the references (see Howell, Barden, and Lo [1997]). Instead, let us turn our attention to the trajectory correction maneuver (TCM) problem.

\subsection{The TCM Problem}

Genesis will be launched from a Delta 7326 launch vehicle (L/V) using a Thiakol Star37 motor as the final upper stage. The most important error introduced by the inaccuracies of the launch vehicle is the velocity magnitude error. In this case, the expected error is $7 \mathrm{~m} / \mathrm{s}$ ( 1 sigma value) relative to a boost of approximately 3200 $\mathrm{m} / \mathrm{s}$ from a $200 \mathrm{~km}$ circular altitude Earth orbit. In the space industry, we call the change in velocity a $\Delta V$. It is typical in space missions to use the magnitude of the $\Delta V$ as a measure of the spacecraft performance. The propellant mass is a much less stable quantity as a measure of spacecraft performance, since it is dependent on the spacecraft mass and various other parameters which change frequently as the spacecraft is being built.

Although a $7 \mathrm{~m} / \mathrm{s}$ error for a $3200 \mathrm{~m} / \mathrm{s}$ maneuver may seem rather small, it actually is considered quite large. Unfortunately, one of the characteristics of halo orbit missions is that, unlike interplanetary mission launches, they are extremely sensitive to launch errors. Typical interplanetary launches can correct launch vehicle errors 7 to 14 days after the launch. In contrast, halo orbit missions must generally correct the launch error within the first day after launch, due to energy concerns. This critical Trajectory Correction Maneuver is called TCM1, being the first TCM of any mission. Two clean up maneuvers, TCM2 and TCM3, generally follow TCM1 after a week or more, depending on the situation. 
From the exuation for a conic: orbit,

$$
E=\frac{V^{2}}{2}-\frac{G m}{R}
$$

where $E$ is Keplerian energy, $V$ is velocity, $G m$ is the gravitational mass, and $R$ is the position, it can be seen that

$$
\delta V=\frac{\delta E}{V}
$$

where $\delta V$ and $\delta E$ denote the variations in velocity and energy, respectively. In particular, for highly elliptical orbits, $V$ decreases sharply as a function of time past perigee. Hence, the correction maneuver, $\Delta V$, grows sharply in inverse proportion to the time from launch. For a large launch vehicle error, which is possible in the case of Genesis, the correction maneuver TCM1 can quickly grow beyond the capability of the spacecraft's propulsion system.

The Genesis spacecraft, built in the spirit of NASA's new low cost mission approach, is very basic. This makes the execution of an early TCM1 extremely difficult and risky. It is highly desirable to delay TCM1 by as long as possible, even at the expense of expenditure of the precious $\Delta V$ budget. In fact, the Genesis Project would prefer TCM1 be performed at 2 to 7 days after launch, or later if at all possible. The design of the current Genesis TCM1 retargets the state after launch back to the nominal HOI state (see Lo, Williams et al [1998]). This approach is based on linear analysis and is perfectly adequate if TCM1 is performed within 24 hours after launch. Beyond launch +24 hours, the correction cost can become prohibitively high. See also Wilson, Howell, and Lo [1999] for another approach to targeting that may be applicable for Genesis.

The desire to increase the time between launch and TCM1 suggests that one use a nonlinear approach, combining dynamical systems theory with optimal control techniques. We explore two similar but slightly different approaches and are able to obtain in both cases an optimal maneuver strategy that fits within the Genesis $\Delta V$ budget of $150 \mathrm{~m} / \mathrm{s}$ for the transfer portion of the trajectory. These are:

1. HOI technique: use optimal control techniques to retarget the halo orbit with the original nominal trajectory as the initial guess.

2. MOI technique: target the stable manifold.

Both methods are shown to yield good results.

\section{Optimal Control for Trajectory Correction Maneu- vers}

We now introduce the general problem of optimal control for dynamical systems. We start by recasting the TCM problem as a spacecraft trajectory planning problem. Mathematically they are exactly the same. We discuss the spacecraft trajectory 
plaming problem as an optimization problem and highlight the formulation characteristics and particular solution requirements. Then the fuel efficiency caused by possible perturbation in the launch velocity and by different delays in TCM1 is exactly the sensitivity analysis of the optimal solution. COOPT, the software we use, is an excellent tool in solving this type of problem, both in providing a solution for the trajectory planning problem with optimal control and in studying the sensitivity of different parameters.

We emphasize that the objective in this work is not to design the transfer trajectory, but rather to investigate recovery issues related to possible launch velocity errors. We therefore assume that a nominal transfer trajectory (corresponding to zero errors in launch velocity) is available. For the nominal trajectory in our numerical experiments in this paper, we do not use the actual Genesis mission transfer trajectory, but rather an approximation obtained with a more restricted model. It has been shown elsewhere (for example, Howell, Barden, and Lo [1997]) that the general qualitative characteristics found in the restricted models translate well when extended into more accurate models; we expect the same correlation with this work as well.

\subsection{Recasting TCM as a Trajectory Planning Problem}

Although different from a dynamical systems perspective, the HOI and MOI problems are very similar once cast as optimization problems. In the HOI problem, a final maneuver (jump in velocity) is allowed at $T_{\mathrm{HOI}}=t_{\max }$, while in the MOI problem, the final maneuver takes place on the stable manifold at $T_{\mathrm{MOI}}<t_{\max }$ and no maneuver is allowed at $T_{\mathrm{HOI}}=t_{\max }$. A halo orbit insertion trajectory design problem can be simply posed as:

Find the maneuver times and sizes to minimize fuel consumption $(\Delta V)$ for a trajectory starting near Earth and ending on the specified halo orbit around the Lagrange point $L_{1}$ of the Sun-Earth system at a position and velocity state consistent with the HOI time.

The optimization problem as stated has two important features. First, it involves discontinuous controls, since the impulsive maneuvers are represented by jumps in the velocity of the spacecraft. A reformulation of the problem to cast it into the framework required by continuous optimal control algorithms will be discussed later in this section. Secondly, the final halo orbit insertion time $T_{\mathrm{HOI}}$, as well as all intermediate maneuver times, must be included among the optimization parameters (p). This too requires further reformulation of the dynamical model to capture the influence of these parameters on the solution at a given optimization iteration.

Next, we discuss the reformulations required to solve the HOI discontinuous control problem; modifications of the following procedure required to solve the MOI problem are discussed in $\S 3.2$. We assume that the evolution of the trajectory is described by a generic set of six ODEs

$$
\mathbf{x}^{\prime}=\mathbf{f}(t, \mathbf{x})
$$




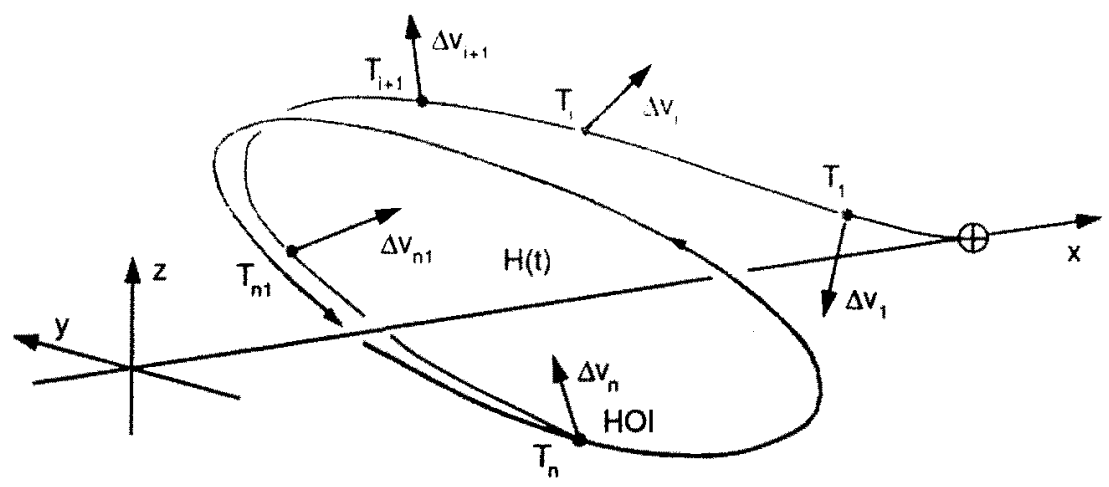

Figure 2: Transfer trajectory. Maneuvers take place at times $T_{i}, i=1,2, \ldots, n$. In the stable manifold insertion problem, there is no maneuver at $T_{n}$, i.e. $\Delta \mathbf{v}_{n}=0$.

where $\mathbf{x}=\left(\mathbf{x}^{p} ; \mathbf{x}^{v}\right) \in \mathbb{R}^{6}$ contains both positions $\left(\mathbf{x}^{p}\right)$ and velocities $\left(\mathbf{x}^{v}\right)$. The dynamical model of Equation (3) can be either the CRTBP or a more complex model that incorporates the influence of the Moon and other planets. In this paper, we use the CRTBP approximation; other models will be investigated in future work.

To deal with the discontinuous nature of the impulsive control maneuvers, the equations of motion (e.o.m.) are solved simultaneously on each interval between two maneuvers. Let the maneuvers $M_{1}, M_{2}, \ldots, M_{n}$ take place at times $T_{i}, i=1,2, \ldots, n$ and let $\mathbf{x}_{i}(t), t \in\left[T_{i-1}, T_{i}\right]$ be the solution of Equation (3) on the interval $\left[T_{i-1}, T_{i}\right]$ (see Figure 2).

To capture the influence of the maneuver times on the solution of the e.o.m. and to be able to solve the e.o.m. simultaneously, we scale the time in each interval by the duration $\Delta T_{i}=T_{i}-T_{i-1}$. As a consequence, all time derivatives in Equation (3) are scaled by $1 / \Delta T_{i}$. The dimension of the dynamical system is thus increased to $N_{x}=6 n$.

Position continuity constraints are imposed at each maneuver, that is,

$$
\mathbf{x}_{i}^{p}\left(T_{i}\right)=\mathbf{x}_{i+1}^{p}\left(T_{i}\right), \quad i=1,2, \ldots, n-1 .
$$

In addition, the final position is forced to lie on the halo orbit (or stable manifold), that is,

$$
\mathbf{x}_{n}^{p}\left(T_{n}\right)=\mathbf{x}_{H}^{p}\left(T_{n}\right)
$$

where the halo orbit is parameterized by the HOI time $T_{n}$. Additional constraints dictate that the first maneuver (TCM1) is delayed by at least a prescribed amount $T C M 1_{\min }$, that is,

$$
T_{1} \geq T C M 1_{\min }
$$

and that the order of maneuvers is respected,

$$
T_{i-1}<T_{i}<T_{i+1}, \quad i=1,2, \ldots, n-1 .
$$


With a cost fumetion tefined as some moasure of the velocity discontimuties

$$
\begin{aligned}
& \Delta \mathbf{v}_{i}=\mathbf{x}_{i+i}^{\prime \prime}\left(T_{i}\right)-\mathbf{x}_{i}^{\prime \prime}\left(T_{i}\right), \quad i=1,2, \ldots, n-1, \\
& \Delta \mathbf{v}_{n}=\mathbf{x}_{H}^{\prime \prime}\left(T_{n}\right)-\mathbf{x}_{n}^{v}\left(T_{n}\right),
\end{aligned}
$$

the optimization problem becomes

$$
\min _{T_{i}, \mathbf{x}_{i}, \Delta \mathbf{v}_{i}} C\left(\Delta \mathbf{v}_{i}\right)
$$

subject to the constraints in Equations (4)-(8). More details on selecting the form of the cost function are given in $\S 3$.

\subsection{Launch Errors and Sensitivity Analysis}

In many optimal control problems, obtaining an optimal solution is not the only goal. The influence of problem parameters on the optimal solution (the so called sensitivity of the optimal solution) is also needed. Sensitivity information provides a first-order approximation to the behavior of the optimal solution when parameters are not at their optimal values or when constraints are slightly violated.

In the problems treated in this paper, for example, we are interested in estimating the changes in fuel efficiency $(\Delta V)$ caused by possible perturbations in the launch velocity $\left(\epsilon_{0}^{v}\right)$ and by different delays in the first maneuver (TCM1). As we show in $\S 3$, the cost function is very close to being linear in these parameters $\left(T C M 1_{\min }\right.$ and $\left.\epsilon_{0}^{v}\right)$. Therefore, evaluating the sensitivity of the optimal cost is a very inexpensive and accurate method of assessing the influence of different parameters on the optimal trajectory (especially in our problem).

In COOPT, we make use of the Sensitivity Theorem (see Bertsekas [1995]) for nonlinear programming problems with equality and/or inequality constraints:

Theorem 2.1 Let $f, h$, and $g$ be twice continuously differentiable and consider the family of problems

$$
\begin{aligned}
& \text { minimize } f(x) \\
& \text { subject to } h(x)=u, \quad g(x) \leq v,
\end{aligned}
$$

parameterized by the vectors $u \in \mathbb{R}^{m}$ and $v \in \mathbb{R}^{r}$. Assume that for $(u, v)=(0,0)$ this problem has a local minimum $x^{*}$, which is regular and which together with its associated Lagrange multiplier vectors $\lambda^{*}$ and $\mu^{*}$, satisfies the second order sufficiency conditions. Then there exists an open sphere $S$ centered at $(u, v)=(0,0)$ such that for every $(u, v) \in S$ there is an $x(u, v) \in \mathbb{R}^{n}, \lambda(u, v) \in \mathbb{R}^{m}$, and $\mu(u, v) \in \mathbb{R}^{r}$, which are a local minimum and associated Lagrange multipliers of problem (10). Furthermore, $x(\cdot), \lambda(\cdot)$, and $\mu(\cdot)$ are continuously differentiable in $S$ and we have $x(0,0)=x^{*}, \lambda(0,0)=\lambda^{*}, \mu(0,0)=\mu^{*}$. In addition, for all $(u, v) \in S$, there holds

$$
\begin{aligned}
& \nabla_{u} p(u, v)=-\lambda(u, v), \\
& \nabla_{v} p(u, v)=-\mu(u, v),
\end{aligned}
$$

where $p(u, v)$ is the optimal cost parameterized by $(u, v)$,

$$
p(u, v)=\cdot f(x(u, v))
$$


The influence of delaying the maneuver TCM1 is thus directly computed from the Lagrange multiplier associated with the constraint of Equation (6). To evaluate sensitivities of the cost function with respect to perturbations in the launch velocity $\left(\epsilon_{0}^{v}\right)$, we must include this perturbation explicitly as an optimization parameter and fix it to some prescribed value through an equality constraint. That is, the launch velocity is set to

$$
\mathbf{v}(0)=\mathbf{v}_{0}^{\text {nom }}\left(1+\frac{\epsilon_{0}^{v}}{\left\|\mathbf{v}_{0}^{\text {nom }}\right\|}\right)
$$

where $\mathbf{v}_{0}^{\text {nom }}$ is the nominal launch velocity and

$$
\epsilon_{0}^{v}=\epsilon
$$

for a given $\epsilon$. The Lagrange multiplier associated with the constraint in Equation (14) yields the desired sensitivity.

\subsection{Description of the COOPT Software}

COOPT is a software package for optimal control and optimization of systems modeled by differential-algebraic equations (DAE) (see Brenan, Campbell and Petzold [1995]), developed by the Computational Science and Engineering Group at the University of California, Santa Barbara. It has been designed to control and optimize a general class of DAE systems, which may be quite large. Here we describe the basic methods used in COOPT. We consider the DAE system

$$
\begin{array}{r}
\mathbf{F}\left(t, \mathbf{x}, \mathbf{x}^{\prime}, \mathbf{p}, \mathbf{u}(t)\right)=\mathbf{0} \\
\mathbf{x}\left(t_{1}, \mathbf{r}\right)=\mathbf{x}_{1}(\mathbf{r})
\end{array}
$$

where the DAE is index zero, one, or semi-explicit index two (see Ascher and Petzold [1998], or Brenan, Campbell, and Petzold [1995]) and the initial conditions have been chosen so that they are consistent (that is, the constraints of the DAE are satisfied). The control parameters $\mathbf{p}$ and $\mathbf{r}$ and the vector-valued control function $\mathbf{u}(t)$ must be determined such that the objective function

$$
\int_{t_{1}}^{t_{\max }} \Psi(t, \mathbf{x}(t), \mathbf{p}, \mathbf{u}(t)) d t+\Theta\left(t_{\max }, \mathbf{x}\left(t_{\max }\right), \mathbf{p}, \mathbf{r}\right),
$$

is minimized and some additional inequality constraints

$$
\mathbf{g}(t, \mathbf{x}(t), \mathbf{p}, \mathbf{u}(t)) \geq 0
$$

are satisfied. The optimal control function $\mathbf{u}^{*}(t)$ is assumed to be continuous. To represent $\mathbf{u}(t)$ in a low-dimensional vector space, we use piecewise polynomials on $\left[t_{1}, t_{\max }\right]$, where their coefficients are determined by the optimization. For ease of presentation we can therefore assume that the vector $\mathbf{p}$ contains both the parameters and these coefficients (we let $N_{p}$ denote the combined number of these values) and discard the control function $\mathbf{u}(t)$ in the remainder of this section. Also, we consider 
that the initial states are fixed and therefore discard the dependency of $x_{1}$ on $\mathbf{r}$. Hence, we consider

$$
\begin{aligned}
& \mathbf{F}\left(t, \mathbf{x}, \mathbf{x}^{\prime}, \mathbf{p}\right)=0, \quad \mathbf{x}\left(t_{1}\right)=\mathbf{x}_{1}, \\
& \int_{t_{1}}^{t_{\max }} \psi(t, \mathbf{x}(t), \mathbf{p}) d t+\Theta\left(t_{\max }, \mathbf{x}\left(t_{\max }\right), \mathbf{p}\right) \quad \text { is minimized, } \\
& \mathbf{g}(t, \mathbf{x}(t), \mathbf{p}) \geq 0 .
\end{aligned}
$$

There are a number of well-known methods for direct discretization of the optimal control problem in Equations (18), for the case in which the DAEs can be reduced to ordinary differential equations (ODEs) in standard form. COOPT implements the single shooting method and a modified version of the multiple shooting method, both of which allow the use of adaptive DAE software.

In the multiple shooting method, the time interval $\left[t_{1}, t_{\max }\right]$ is divided into subintervals $\left[t_{i}, t_{i+1}\right]\left(i=1, \ldots, N_{t x}\right)$, and the differential equations in Equation (18a) are solved over each subinterval, where additional intermediate variables $\mathbf{X}_{i}$ are introduced. On each subinterval we denote the solution at time $t$ of Equation (18a) with initial value $\mathbf{X}_{i}$ at $t_{i}$ by $\mathbf{x}\left(t, t_{i}, \mathbf{X}_{i}, \mathbf{p}\right)$.

Continuity between subintervals in the multiple shooting method is achieved via the continuity constraints

$$
\mathbf{C}_{1}^{i}\left(\mathbf{X}_{i+1}, \mathbf{X}_{i}, \mathbf{p}\right) \equiv \mathbf{X}_{i+1}-\mathbf{x}\left(t_{i+1}, t_{i}, \mathbf{X}_{i}, \mathbf{p}\right)=\mathbf{0}
$$

The additional constraints of Equation (18c) are required to be satisfied at the boundaries of the shooting intervals

$$
\mathbf{C}_{2}^{i}\left(\mathbf{X}_{i}, \mathbf{p}\right) \equiv \mathbf{g}\left(t_{i}, \mathbf{X}_{i}, \mathbf{p}\right) \geq \mathbf{0}
$$

Following common practice, we write

$$
\Phi(t)=\int_{t_{1}}^{t} \psi(\tau, \mathbf{x}(\tau), \mathbf{p}) d \tau
$$

which satisfies $\Phi^{\prime}(t)=\psi(t, \mathbf{x}(t), \mathbf{p}), \Phi\left(t_{1}\right)=0$. This introduces another equation and variable into the differential system in Equation (18a). The discretized optimal control problem becomes

$$
\min _{\mathbf{x}_{2}, \ldots, \mathbf{X}_{N t x}, \mathbf{p}} \Phi\left(t_{\max }\right)+\Theta\left(t_{\max }\right)
$$

subject to the constraints

$$
\begin{array}{r}
\mathrm{C}_{1}^{i}\left(\mathbf{X}_{i+1}, \mathbf{X}_{i}, \mathbf{p}\right)=0 \\
\mathbf{C}_{2}^{i}\left(\mathbf{X}_{i}, \mathbf{p}\right) \geq \mathbf{0}
\end{array}
$$

This problem can be solved by an optimization algorithm. We use the solver SNOPT (see Gill, Murray and Saunders [1997]), which incorporates a sequential 


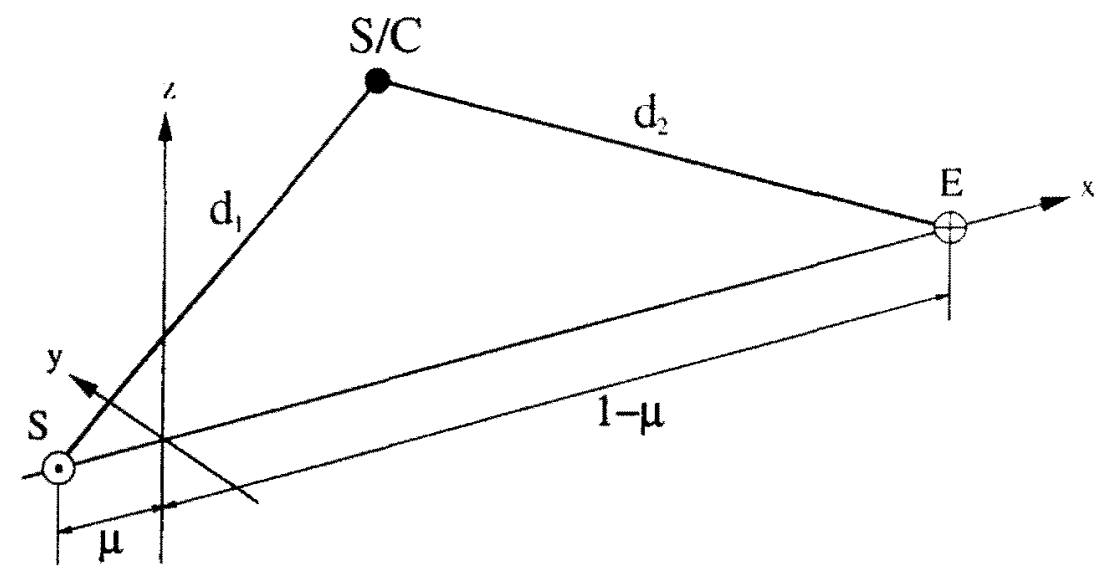

Figure 3: Coordinate frame in the CRTBP approximation.

quadratic programming (SQP) method (see Gill, Murray and Wright [1981]). The SQP methods require a gradient and Jacobian matrix that are the derivatives of the objective function and constraints with respect to the optimization variables. We compute these derivatives via DAE sensitivity software DASPK3.0 ( $\mathrm{Li}$ and Petzold [1999]). The sensitivity equations to be solved by DASPK 3.0 are generated via the automatic differentiation software ADIFOR (see Bischof, Carle, Corliss, Griewank and Hovland [1997]).

This basic multiple-shooting type of strategy can work very well for small-tomoderate size ODE systems, and has an additional advantage that it is inherently parallel. However, for large-scale ODE and DAE systems there is a problem because the computational complexity grows rapidly with the dimension of the ODE system. COOPT implements a highly efficient modified multiple shooting method (Petzold, Rosen, Gill, Jay and Park [1992] and Serban [1999]) which reduces the computational complexity to that of single shooting for large-scale problems. However, we have found it sufficient to use single shooting for the trajectory design problems treated in this paper.

\section{Numerical Results}

Circular Restricted Three-Body Model. As mentioned earlier, we use the equations of motion derived under the CRTBP assumption as the underlying dynamical model in Equation (3). In this model, it is assumed that the primaries (Earth and Sun in our case) move on circular orbits around the center of mass of the system and that the third body (the spacecraft) does not influence the motion of the primaries. We write the equations in a rotating frame, as in Figure 3. 
Using nondimensional units, the equations of motion in the CRTBP model are

$$
\begin{aligned}
& \dot{x}_{1}=x_{1} \\
& \dot{x}_{2}=x_{5} \\
& \dot{x}_{3}=x_{6} \\
& \dot{x}_{4}=2 x_{2}+\frac{\partial U}{\partial x_{1}} \\
& \dot{x}_{5}=-2 x_{1}+\frac{\partial U}{\partial x_{2}} \\
& \dot{x}_{6}=\frac{\partial U}{\partial x_{3}}
\end{aligned}
$$

where

$$
\begin{aligned}
\mathbf{x} & =\left[x_{1}, x_{2}, x_{3}, x_{4}, x_{5}, x_{6}\right]^{T}=\left[x, y, z, v_{x}, v_{y}, v_{z}\right]^{T} \\
U & =\frac{1}{2}\left(x_{1}^{2}+x_{2}^{2}\right)+\frac{1-\mu}{d_{1}}+\frac{\mu}{d_{2}} \\
d_{1} & =\left(\left(x_{1}+\mu\right)^{2}+x_{2}^{2}+x_{3}^{2}\right)^{1 / 2} \\
d_{2} & =\left(\left(x_{1}-1+\mu\right)^{2}+x_{2}^{2}+x_{3}^{2}\right)^{1 / 2}
\end{aligned}
$$

and $\mu$ is the ratio between the mass of the Earth and the mass of the Sun-Earth system,

$$
\mu=\frac{m_{\oplus}}{m_{\oplus}+m_{\odot}}
$$

where $\oplus$ denotes the Earth and $\odot$ the Sun. In the above equations, time is scaled by the period of the primaries orbits $(T / 2 \pi$, where $T=1$ year $)$, positions are scaled by the Sun-Earth distance $\left(L \equiv d_{\oplus \odot}=1.49597927 \cdot 10^{8} \mathrm{~km}\right)$, and velocities are scaled by the Earth's average orbital speed around the Sun $(2 \pi L / T=29.80567 \mathrm{~km} / \mathrm{s})$.

\section{Choice of Cost Function.}

At this point we need to give some more details on the choice of an appropriate cost function for the optimization problem (9). Typically in space missions, the spacecraft performance is measured in terms of the maneuver sizes $\Delta \mathbf{v}_{i}$. We consider the following two cost functions.

$$
C_{1}(\Delta \mathbf{v})=\sum_{i=1}^{n}\left\|\Delta \mathbf{v}_{i}\right\|^{2}
$$

and

$$
C_{2}(\Delta \mathbf{v})=\sum_{i=1}^{n}\left\|\Delta \mathbf{v}_{i}\right\| .
$$

While the second of these may seem physically the most meaningful, as it measures the total sum of the maneuver sizes, such a cost function is nondifferentiable whenever one of the maneuvers vanishes. In our case, this problem occurs already at the 
first optimization iteration, as the initial guess transfer trajectory only has a single nonzero maneuver at halo insertion. The first cost function, on the other hand, is differentiable everywhere.

Although the cost function $C_{\mathrm{L}}$ is more appropriate for the optimizer, it raises two new problems. Not only is it not as physically meaningful as the cost function $C_{2}$, but, in some particular cases, decreasing $C_{1}$ may actually lead to increases in $\mathrm{C}_{2}$.

To resolve these issues, we use the following three-stage optimization sequence:

1. Starting with the nominal transfer trajectory as initial guess, and allowing initially $n$ maneuvers, we minimize $C_{1}$ to obtain a first optimal trajectory, $\mathcal{T}_{1}^{*}$.

2. Using $\mathcal{T}_{1}^{*}$ as initial guess, we minimize $C_{2}$ to obtain $\mathcal{T}_{2}^{*}$. It is possible that during this optimization stage some maneuvers can become very small. After each optimization iteration we monitor the feasibility of the iterate and the sizes of all maneuvers. As soon as at least one maneuver decreases under a prescribed threshold (typically $0.1 \mathrm{~m} / \mathrm{s}$ ) at some feasible configuration, we stop the optimization algorithm.

3. If necessary, a third optimization stage, using $\mathcal{T}_{2}^{*}$ as initial guess and $C_{2}$ as cost function is performed with a reduced number of maneuvers $\bar{n}$ (obtained by removing those maneuvers identified as "zero maneuvers" in step 2).

Merging Optimal Control with Dynamical Systems Theory Next, we present results for the halo orbit insertion problem $(\S 3.1)$ and for the stable manifold insertion problem ( $\S 3.2)$. In both cases we are investigating the effect of varying times for $T C M 1_{\min }$ on the optimal trajectory, for given perturbations in the nominal launch velocity. The staggered optimization procedure described above is applied for values of $T C M 1_{\min }$ ranging from 1 day to 5 days and perturbations in the magnitude of the launch velocity $\epsilon_{0}^{v}$ ranging from $-7 \mathrm{~m} / \mathrm{s}$ to $+7 \mathrm{~m} / \mathrm{s}$. We present typical transfer trajectories, as well as the dependency of the optimal cost on the two parameters of interest. In addition, using the algorithm presented in $\S 2.2$, we perform a sensitivity analysis of the optimal solution. For the Genesis TCM problem it turns out that sensitivity information of first order is sufficient to characterize the influence of $T C M 1_{\min }$ and $\epsilon_{0}^{v}$ on the spacecraft performance.

The merging of optimal control and dynamical systems has been done through either (1) the use of the nominal transfer trajectory as a really accurate initial guess, or (2) the use of the stable invariant manifold.

\subsection{Halo Orbit Insertion (HOI) Problem}

In this problem we directly target the selected halo orbit with the last maneuver taking place at the HOI point. Using the optimization procedure described in the previous section, we compute the optimal cost transfer trajectories for various combinations of $T C M 1_{\min }$ and $\epsilon_{0}^{v}$. In all of our computations, the launch conditions are those corresponding to the nominal transfer trajectory, i.e., 


$$
\begin{aligned}
x_{(1)}^{\text {nlm }} & =1.496032475412839 \cdot 10^{8} \mathrm{~km} \\
y_{0}^{\text {nom }} & =1.943203061350240 \cdot 10^{3} \mathrm{~km} \\
z_{0}^{\text {nom }} & =-2.479095822700627 \cdot 10^{3} \mathrm{~km} \\
\left(v_{0}^{\text {nom }}\right)_{x} & =-4.612683390613825 \mathrm{~km} / \mathrm{s} \\
\left(v_{0}^{\text {nom }}\right)_{y} & =9.412034579485869 \mathrm{~km} / \mathrm{s} \\
\left(v_{0}^{\text {nom }}\right)_{z} & =-3.479627336419212 \mathrm{~km} / \mathrm{s}
\end{aligned}
$$

with the launch velocity perturbed as described in $\S 2.2$. These initial conditions are given in the Earth-Sun barycentered rotating frame.

As an example, we present complete results for the case in which the launch velocity is perturbed by $-3 \mathrm{~m} / \mathrm{s}$ and the first maneuver correction is delayed by at least 3 days. Initially, we allow for $n=4$ maneuvers. In the first optimization stage, the second type of cost function has a value of $C_{1}^{*}=1153.998(\mathrm{~m} / \mathrm{s})^{2}$ after 5 iterations. This corresponds to $C_{2}^{*}=50.9123 \mathrm{~m} / \mathrm{s}$. During the second optimization stage, we monitor the sizes of all four maneuvers, while minimizing the cost function $C_{1}$. After 23 iterations, the optimization was interrupted at a feasible configuration when at least one maneuver decreased below a preset tolerance of $0.1 \mathrm{~m} / \mathrm{s}$. The corresponding cost function is $C_{2}^{* *}=45.1216 \mathrm{~m} / \mathrm{s}$ with four maneuvers of sizes 33.8252 $\mathrm{m} / \mathrm{s}, 0.0012 \mathrm{~m} / \mathrm{s}, 0.0003 \mathrm{~m} / \mathrm{s}$, and $11.2949 \mathrm{~m} / \mathrm{s}$. In the last optimization stage we remove the second and third maneuvers and again minimize the cost function $C_{2}$. After 7 optimization iterations an optimal solution with $C_{2}^{* * *}=45.0292 \mathrm{~m} / \mathrm{s}$ is obtained. The two maneuvers of the optimal trajectory have sizes of $33.7002 \mathrm{~m} / \mathrm{s}$ and $11.3289 \mathrm{~m} / \mathrm{s}$ and take place at 3.0000 and 110.7969 days after launch, respectively. The resulting optimal trajectory is presented in Figure 4.

Lagrange multipliers associated with the constraints of Equations (6) and (14) give the sensitivities of the optimal solution with respect to launching velocity perturbation, $-10.7341(\mathrm{~m} / \mathrm{s}) /(\mathrm{m} / \mathrm{s})$, and delay in first maneuver correction, 4.8231 $(\mathrm{m} / \mathrm{s}) /$ days.

Launch Errors and Sensitivity Analysis The staggered optimization procedure was then applied for all values of $T C M 1_{\min }$ and $\epsilon_{0}^{v}$ in the regime of interest. In a first experiment, we investigate the possibility of correcting for errors in the launch velocity using at most two maneuvers $(n=2)$. The surface of optimal cost $\left(C_{2}\right.$ in $\mathrm{m} / \mathrm{s}$ ) as a function of these two parameters is presented in Figure 5. Numerical values are given in Table 1.

Except for the cases in which there is no error in the launch velocity (and for which the final optimal transfer trajectories have only one maneuver at $\mathrm{HOI}$ ), the first correction maneuver is always on the prescribed lower bound $T C M 1_{\min }$. The evolution of the time at which the halo insertion maneuver takes place as a function of the two parameters considered is shown in Figure 6. 


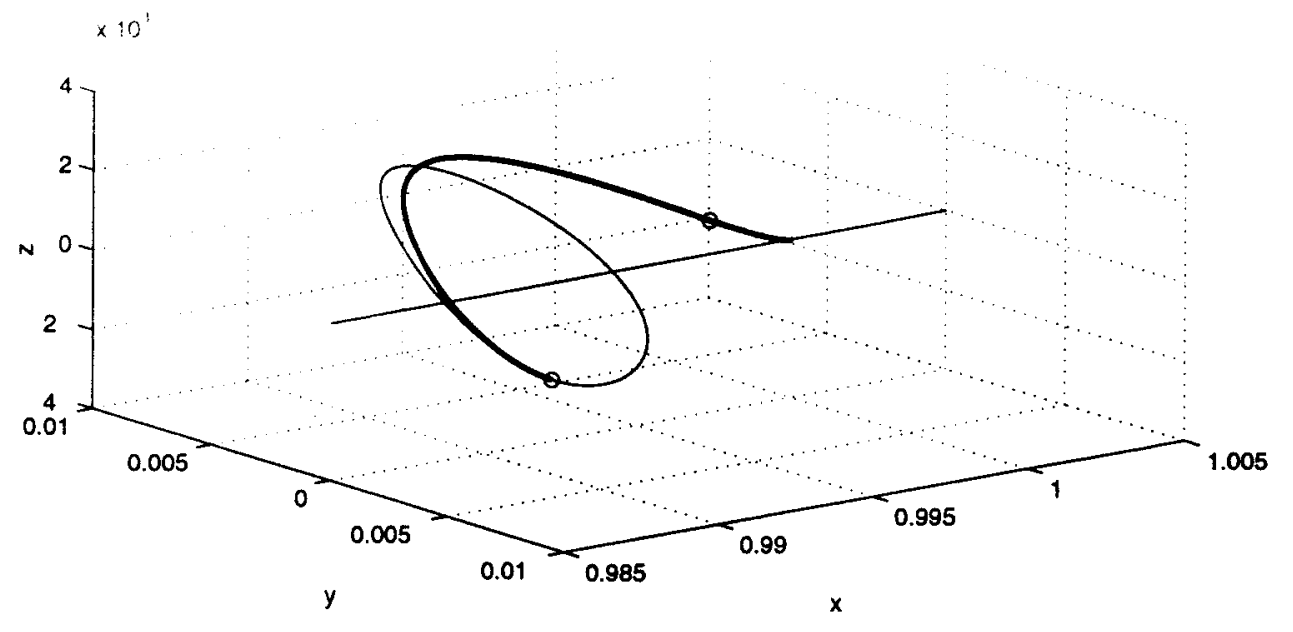

Figure 4: HOI problem. Optimal transfer trajectory for $T C M 1_{\min }=4$ days, $\epsilon_{0}^{v}=3 \mathrm{~m} / \mathrm{s}$, and $n=4$. The optimal trajectory has $\bar{n}=2$ maneuvers (represented by circles).
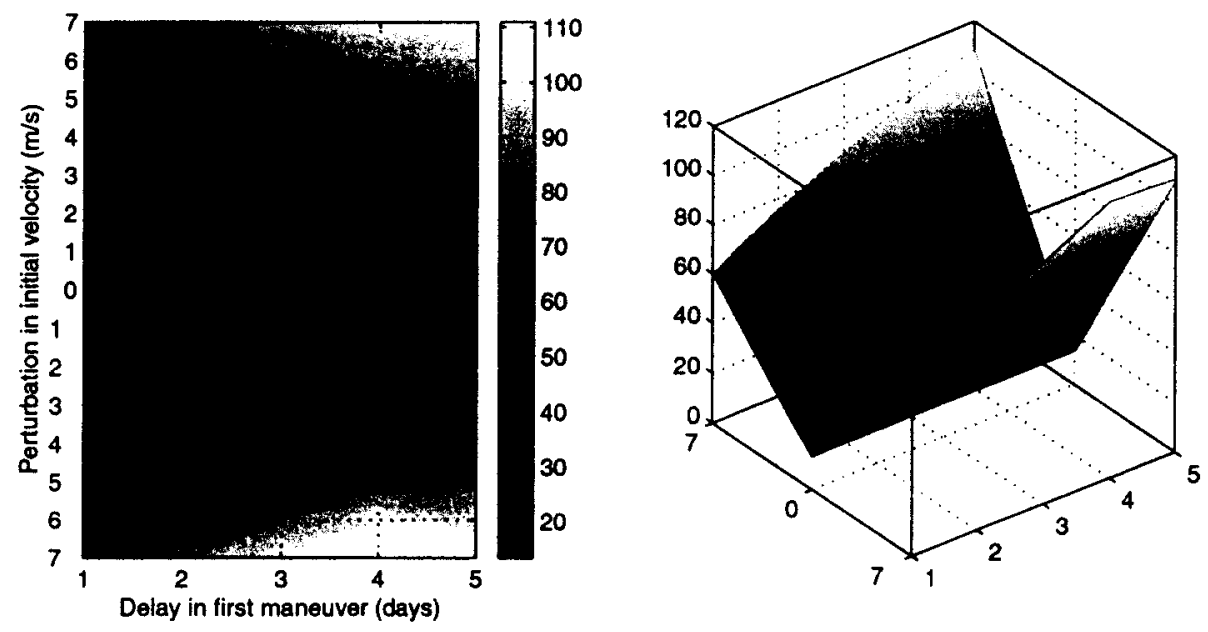

Figure 5: HOI problem. Influence of $T C M 1_{\min }$ and $\epsilon_{0}^{v}$ on the optimal $\operatorname{cost}\left(C_{2}\right.$ in $\left.\mathrm{m} / \mathrm{s}\right)$ for $n=2$. 


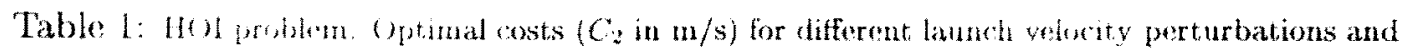
delays in lirst irijoetory corretion maneuver for $n=2$.

\begin{tabular}{|c|c|c|c|c|c|}
\hline \multirow{2}{*}{$\left(\begin{array}{l}7 / 3 / s) \\
(11 / 3)\end{array}\right.$} & \multicolumn{5}{|c|}{ TCMI (days) } \\
\hline & $T$ & 2 & 3 & 4 & 7 \\
\hline-7 & 74.8086 & 76.0845 & 88.4296 & 99.6005 & 109.0305 \\
\hline$i_{z}$ & 54.0461 & 67.0226 & 77.7832 & 86.8630 & 95,3202 \\
\hline .5 & 47.1839 & 57.9451 & 66.6277 & 74.4544 & 81.8284 \\
\hline-4 & 40.2710 & 48.8619 & 55.8274 & 62.0412 & 67.9439 \\
\hline-3 & 33.4476 & 39.8919 & 45.0200 & 49.6804 & 54.1350 \\
\hline-2 & 26.6811 & 30.9817 & 34.3489 & 37.3922 & 40.3945 \\
\hline .1 & 19.9881 & 22.2715 & 23.7848 & 25.2488 & 26.6662 \\
\hline 0 & 13.4831 & 13.3530 & 13.4606 & 13.3465 & 13.2919 \\
\hline i & 23.1900 & 21.9242 & 23.2003 & 24.4154 & 25.5136 \\
\hline 2 & 26.2928 & 30,2773 & 33,3203 & 35.9203 & 38.3337 \\
\hline 3 & 34.6338 & 38.8496 & 43.5486 & 47.7200 & 51.6085 \\
\hline 4 & $\$ 1.4230$ & 47.5266 & 53.0557 & 62.3780 & 68.1411 \\
\hline 3 & 45.9268 & 56.2245 & 64.4292 & 75.0188 & 81.4325 \\
\hline 6 & 53.9004 & 64.9741 & 76.6978 & 83.8795 & 95.2313 \\
\hline 7 & 61.4084 & 75.9169 & 85.4875 & 98.4197 & 106.0411 \\
\hline
\end{tabular}
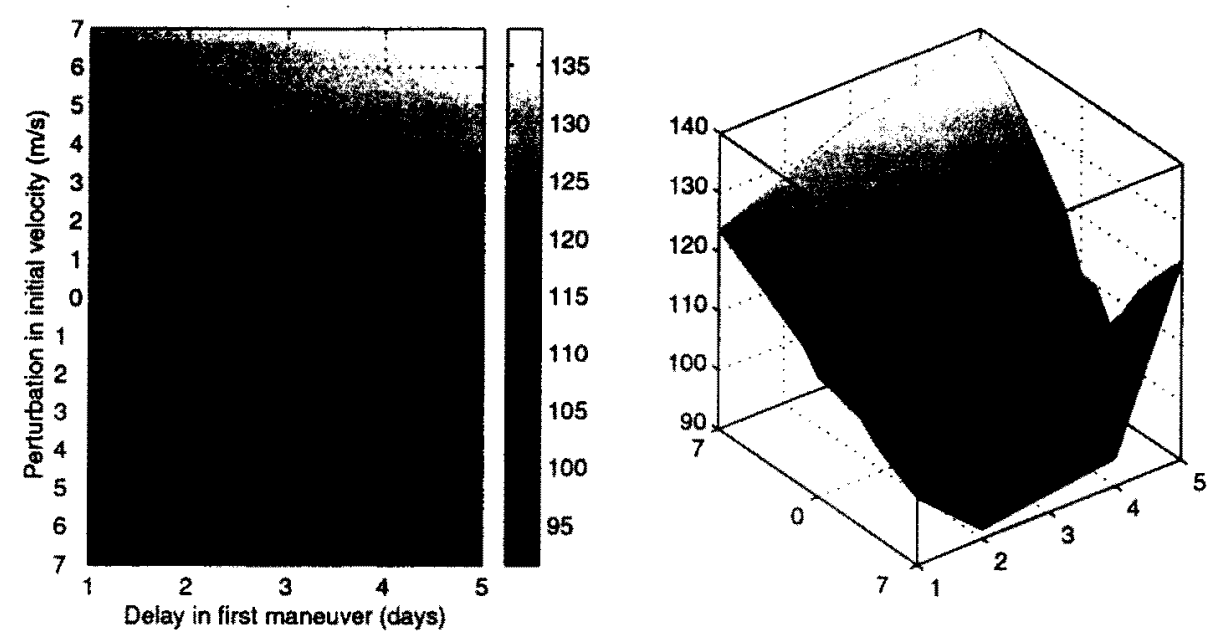

Figure 6: HOI problem. Influence of $T C M 1_{\min }$ and $\epsilon_{0}^{\nu}$ on the halo orbit insertion time $\left(T_{\mathrm{HO}}\right.$ in days) for $n=2$.

Recalling that the nominal transfer trajectory has $T_{\mathrm{HOI}}=110.2$ days, it follows that, for all cases investigated, halo orbit insertion takes place at most 18.6 days earlier or 28.3 days later than in the nominal case.

Several important observations can be drawn from these results. First, it can be seen that, for all cases that we investigated, the optimal costs are well within the $\Delta V$ budget allocated for trajectory correction maneuvers $(150 \mathrm{~m} / \mathrm{s}$ for the Genesis mission). Secondly, as the second plot in Figure 5 shows, the cost function surface is very close to being linear with respect to both $T C M 1_{\min }$ time and launch velocity error. This suggests that first order derivative information, as obtained from sensitivity analysis of the optimal solution $(\S 2.2)$, provides a very good approximation to the surface. For a few points on the cost function surface, we present tangents obtained from sensitivity data in Figure 7. Finally, the halo orbit insertion time is always close enough to that of the nominal trajectory so as to not affect either the collection of the solar wind or the rest of the mission (mainly the duration for which 

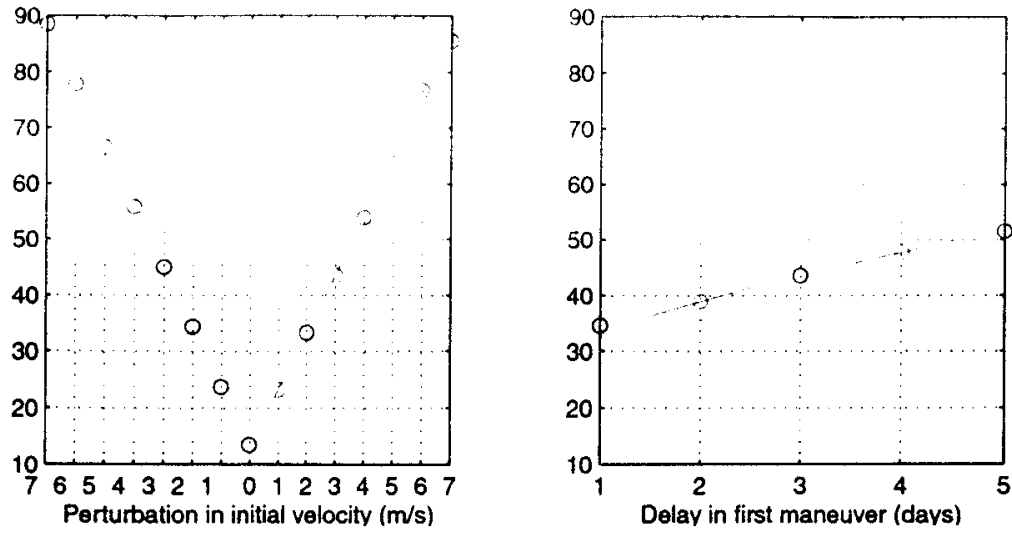

Figure 7: HOI problem. Sensitivity of the optimal solution for $n=2$. Circles correspond to optimization results. Line segments are predictions based on sensitivity computations. The figure on the left was obtained with $T C M 1_{\min }=3$ days and shows the sensitivity of the optimal solution with respect to $\epsilon_{0}^{v}$. The figure on the right was obtained with $\epsilon_{0}^{v}=3 \mathrm{~m} / \mathrm{s}$ and shows the sensitivity of the optimal solution with respect to $T C M 1_{\min }$.

Table 2: HOI problem. Optimal costs $\left(C_{2}\right.$ in $\left.\mathrm{m} / \mathrm{s}\right)$ for different launch velocity perturbations and delays in first trajectory correction maneuver for the best case over $n=2,3,4$.

\begin{tabular}{|c|c|c|c|c|c|}
\hline \multirow{2}{*}{$\begin{array}{c}\epsilon_{0}^{\circ} \\
(\mathrm{m} / \mathrm{s})\end{array}$} & \multicolumn{5}{|c|}{ TCM1 (days) } \\
\hline & 1 & 2 & 3 & 4 & 5 \\
\hline-7 & 61.0946 & 76.0852 & 88.4295 & 99.3123 & 109.9174 \\
\hline-6 & 54.0461 & 67.0212 & 77.7832 & 86.8994 & 95.8202 \\
\hline-5 & 47.1389 & 57.9277 & 66.6277 & 74.4513 & 81.8572 \\
\hline-4 & 40.2710 & 48.8619 & 55.7984 & 62.0398 & 67.9438 \\
\hline-3 & 33.3664 & 39.8919 & 45.0290 & 49.6804 & 54.1357 \\
\hline-2 & 26.6720 & 30.9617 & 34.3489 & 37.3911 & 40.3945 \\
\hline-1 & 19.9674 & 22.1091 & 23.7848 & 25.2640 & 26.6618 \\
\hline 0 & 13.4598 & 13.2902 & 13.4428 & 13.2907 & 13.2919 \\
\hline 1 & 19.8257 & 21.9026 & 23.2005 & 24.4149 & 25.4359 \\
\hline 2 & 26.2933 & 30.2773 & 33.3077 & 35.9203 & 38.3337 \\
\hline 3 & 32.8151 & 38.8496 & 43.5486 & 47.7200 & 51.6085 \\
\hline 4 & 39.3646 & 47.5279 & 53.9557 & 59.7078 & 65.1117 \\
\hline 5 & 45.9127 & 56.2333 & 64.4292 & 71.7790 & 78.7022 \\
\hline 6 & 52.4968 & 64.9741 & 74.9477 & 83.8795 & 92.3090 \\
\hline 7 & 59.0967 & 73.7398 & 85.4875 & 95.9822 & 105.8960 \\
\hline
\end{tabular}

the spacecraft evolves on the halo orbit before initiation of the return trajectory).

In a second set of numerical experiments, we allow initially for as many as $n=4$ maneuvers. This additional degree of freedom in the optimization leads to further reductions in the optimal cost function, as data in Table 2 shows.

The corresponding cost function surface is presented in Figure 8. It is interesting to note that all optimal transfer trajectories have $\bar{n}=2$ maneuvers for negative errors in the launch velocity, $\bar{n}=1$ maneuver if there is no error, and $\bar{n}=3$ maneuvers for positive launch velocity errors. As in the previous case, the time for the first correction maneuver is always on the prescribed lower bound (i.e., $T C M 1=T C M 1_{\min }$ ), while the halo orbit insertion time, shown in Figure 9, is at most 2.6 days earlier or 21.4 days later than in the nominal case. 

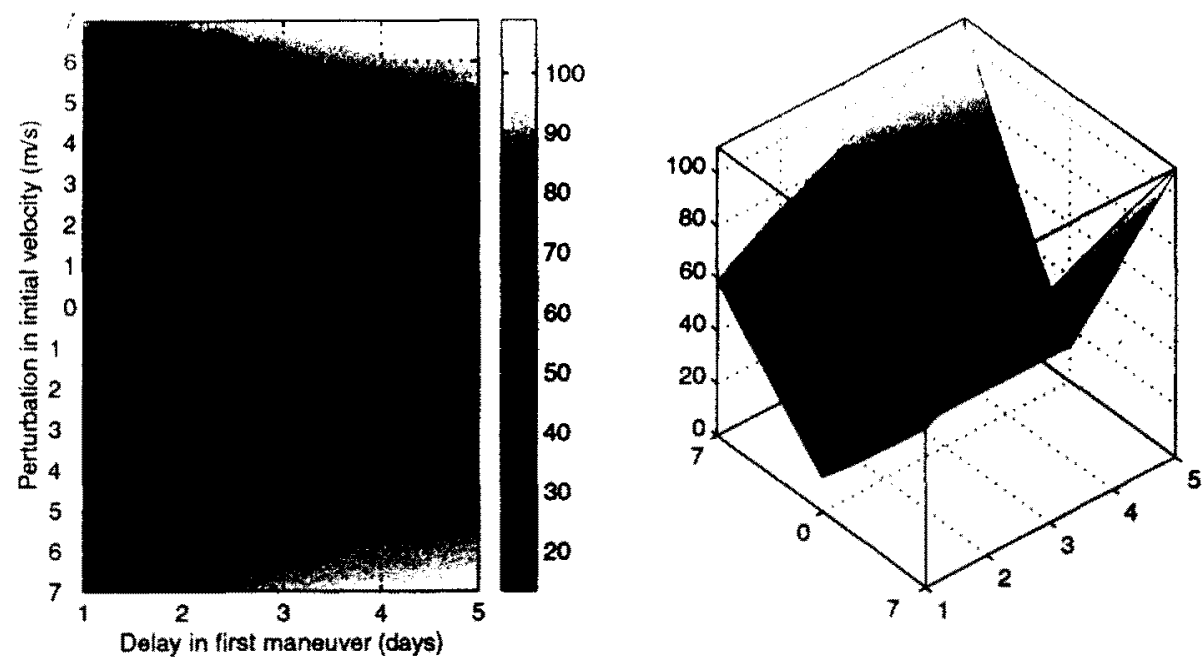

Figure 8: HOI problem. Influence of $T C M 1_{\min }$ and $\epsilon_{0}^{v}$ on the optimal cost $\left(C_{2}\right.$ in m/s). In each case, the best trajectory over $n=2,3,4$ was plotted.
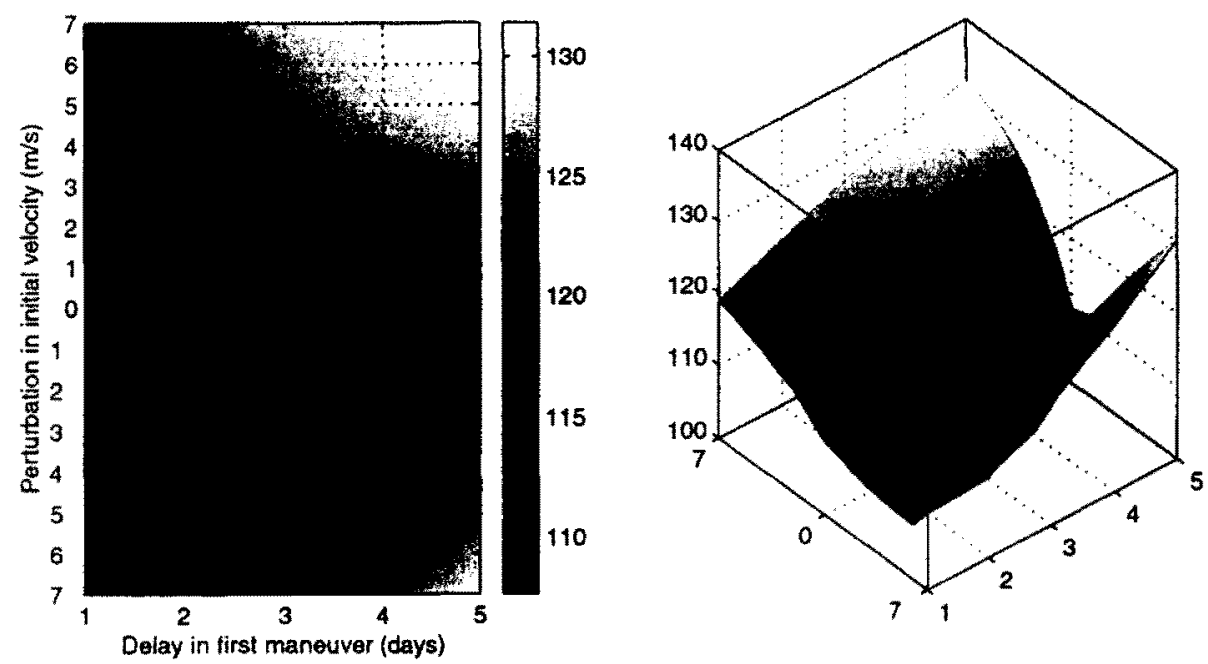

Figure 9: HOI problem. Influence of $T C M 1_{\min }$ and $\epsilon_{0}^{v}$ on the halo orbit insertion time $\left(T_{\mathrm{HO}}\right.$ in days). In each case, the best trajectory over $n=2,3,4$ was plotted. 


\subsection{Stable Manifold Orbit Insertion (MOI) Problem}

Obtaining a Good Initial Guess In the MOI problem the last nonzero maneuver takes place on the stable manifold and there is no maneuver to insert onto the halo orbit. This implies that, in addition to the constraints of Equation (5) imposing that the final position is on the halo orbit, constraints must be imposed to match the final spacecraft velocity with the velocity on the halo orbit. These highly nonlinear constraints, together with the fact that a much larger parameter space is now investigated (we target an entire surface as opposed to just a curve) make the optimization problem much more difficult than the one corresponding to the HOI case. The first problem that arises is that the nominal transfer trajectory is not a good enough initial guess to ensure convergence to an optimum. To obtain an appropriate initial guess we use the following procedure:

1. We start by selecting an HOI time, $T_{\mathrm{HOI}}$. This yields the position and velocity on the halo orbit.

2. The above position and velocity are perturbed in the direction of the stable manifold and the equations of motion in Equation (24) are then integrated backwards in time for a selected duration $T_{S}$. This yields an MOI point which is now fixed in time, position, and velocity.

3. For a given value of $T C M 1_{\min }$ and with $\epsilon_{0}^{v}=0$, and using the nominal transfer trajectory as initial guess, we use COOPT to find a trajectory that targets this MOI point, while minimizing $C_{1}$.

With the resulting trajectory as an initial guess and the desired value of $\epsilon_{0}^{v}$ we proceed with the staggered optimization presented before to obtain the final optimal trajectory for insertion on the stable manifold. During the three stages of the optimization procedure, both the MOI point and the HOI point are free to move (in position, velocity, and time) on the stable manifold surface and on the halo orbit, respectively.

The fact that we are using local optimization techniques implies that the computed optimal trajectories are very sensitive to the choice of the initial guess trajectory. For given values of the problem parameters (such as initial number of maneuvers, perturbation in launch velocity, and lower bound on TCM1) we find optimal trajectories in a neighborhood of the initial guess trajectory. In other words, computed optimal trajectories can be 'steered' towards regions of interest by appropriate choices of initial guess trajectories. For example, taking the launch time to be $T_{L}=0$ and the HOI time $\left(T_{\mathrm{HOI}}^{*}\right)$ of the nominal transfer trajectory as a reference point on the halo orbit, we can investigate a given zone of the design space by an appropriate choice of the HOI point of our initial guess trajectory with respect to $T_{\mathrm{HOI}}^{*}$ (step 1 of the above procedure). That is, we select a value $T_{0}$ such that $T_{\mathrm{HOI}}=T_{\mathrm{HOI}}^{*}+T_{0}$. The point where the initial guess trajectory inserts onto the stable manifold is then defined by selecting the duration $T_{S}$ for which the equations of motion are integrated backwards in time (step 2 of the above procedure). This gives a stable manifold insertion time of $T_{\mathrm{MOI}}=T_{\mathrm{HOI}}-T_{S}=T_{\mathrm{HOI}}^{*}+T_{0}-T_{S}$. Next, 


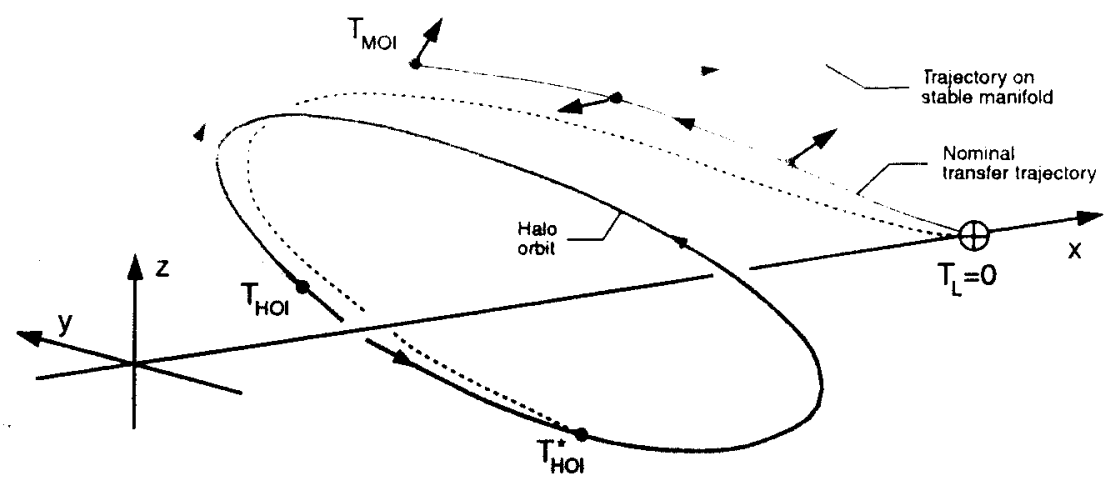

Figure 10: MOI Problem. Description of the initial guess computation procedure.

we use COOPT to evaluate these various choices for the initial guess trajectories (step 3 of the above procedure). A schematic representation of this procedure is shown in Figure 10.

For different combinations of $T_{0}$ and $T_{S}$, Table 3 presents values of $C_{1}^{*}(\Delta \mathbf{v})=$ $\sum_{i=1}^{n}\left\|\Delta \mathbf{v}_{i}\right\|$ corresponding to the optimal initial guess trajectory that targets the resulting MOI point. Note that, for a given value $T_{0}$, there exists a value $T_{S}$ for which we are unable to compute an initial guess trajectory. This is due to the fact that, for these values of $T_{0}$ and $T_{S}$, the resulting $T_{\mathrm{MOI}}$ is too small for COOPT to find a trajectory that targets the MOI point from $T_{L}=0$.

Regions Best Suited for MOI Insertion. From the data given in Table 3 we can identify regions of the stable manifold that are best suited for MOI insertion. Examples of such regions are:

- (Region $A$ ) MOI trajectories that insert to the halo orbit in the same region as the nominal transfer trajectory and which therefore correspond to initial guess trajectories with small $T_{0}$;

- (Region $B$ ) MOI trajectories that have HOI points on the "far side" of the halo orbit and which correspond to initial guess trajectories with halo insertion time around $T_{\mathrm{HOI}}^{*}+1.50\left(T_{0}=1.50 \cdot 365 / 2 \pi=174.27\right.$ days $)$.

These choices are confirmed by the examples from Wilson, Howell, and Lo [1999]. Trajectories in the second region might, at first glance, appear unsuited for the Genesis mission as they would drastically decrease the duration for which the spacecraft evolves on the halo orbit (recall that design of the return trajectory dictates the time at which the spacecraft must leave the halo orbit). However, as the typical MOI trajectory of Figure 11 shows, all trajectories on the stable manifold asymptotically wind onto the halo orbit and are thus very close to the halo orbit for a significant time. This means that collection of solar wind samples can start much earlier than halo orbit insertion, therefore providing enough time for all scientific experiments before the spacecraft leaves the halo orbit. 
Table 3: MOI problem. Initial guess trajectories obtained for different choices of the parameters $T_{0}$ and $T_{S}$. All times are given in nondimensional units.

\begin{tabular}{|c|c|c|c|c|}
\hline$T_{0}$ & Thon & $T_{S}$ & TMOI & $\begin{array}{c}C / \mathrm{s}) \\
(\mathrm{m} / \mathrm{s}\end{array}$ \\
\hline-0.25 & 1.65916 & $\begin{array}{l}0.25 \\
0.50 \\
0.75\end{array}$ & $\begin{array}{c}1.40916 \\
1.15916 \\
-\end{array}$ & $\begin{array}{c}45.7317 \\
93.2419 \\
\end{array}$ \\
\hline 0.00 & 1.90916 & $\begin{array}{l}0.25 \\
0.50 \\
0.75 \\
1.00\end{array}$ & $\begin{array}{c}1.85916 \\
1.40916 \\
1.15916 \\
-\end{array}$ & $\begin{array}{c}21.7515 \\
45.1291 \\
94.0839 \\
-\end{array}$ \\
\hline 0.73 & 2.63016 & $\begin{array}{l}0.25 \\
0.50 \\
0.75 \\
1.00 \\
1.25 \\
1.50 \\
1.73\end{array}$ & $\begin{array}{c}2.40916 \\
2.15916 \\
1.90916 \\
1.65916 \\
1.40916 \\
1.15916 \\
-\end{array}$ & $\begin{array}{c}21.1051 \\
21.4791 \\
24.8072 \\
23.9035 \\
43.2514 \\
86.1323 \\
.\end{array}$ \\
\hline 1.50 & 3.40916 & $\begin{array}{l}0.28 \\
0.50 \\
0.75 \\
1.00 \\
1.25 \\
1.50 \\
1.75 \\
2.00 \\
2.25 \\
2.50 \\
2.75 \\
3.00 \\
3.25\end{array}$ & $\begin{array}{c}3.15916 \\
2.90916 \\
2.65916 \\
2.40916 \\
2.15916 \\
1.90916 \\
1.65916 \\
1.40916 \\
1.13916 \\
0.90916 \\
0.65916 \\
0.40916 \\
-\end{array}$ & $\begin{array}{c}15.9146 \\
16.2152 \\
15.6983 \\
17.6370 \\
27.5903 \\
18.9711 \\
19.4283 \\
28.3686 \\
51.8521 \\
105.7831 \\
212.9097 \\
519.7044 \\
.\end{array}$ \\
\hline
\end{tabular}

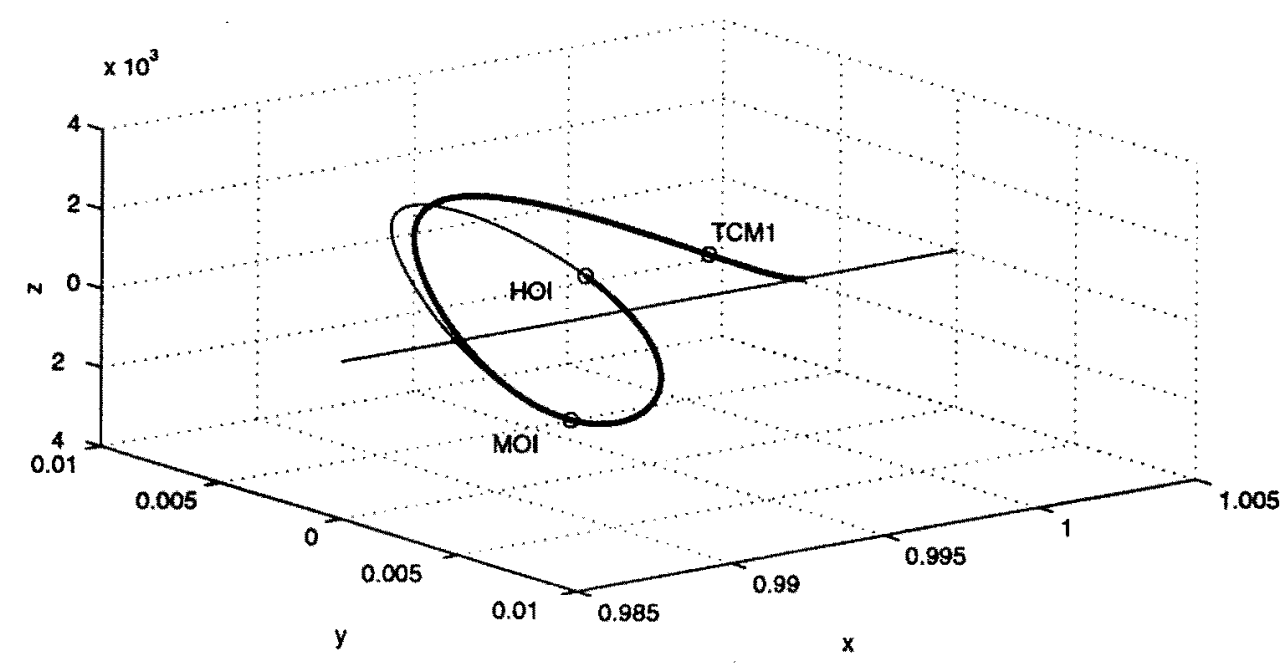

Figure 11: MOI Problem. Optimal transfer trajectory for $T C M 1_{\min }=4$ days, $\epsilon_{0}^{v}=-3 \mathrm{~m} / \mathrm{s}$, and $n=4$. The optimal trajectory has a cost function of $C_{2}=49.1817 \mathrm{~m} / \mathrm{s}$ and $\bar{n}=2$ maneuvers. The first maneuver takes place at $T C M 1=4$ days, the second one at $T_{\mathrm{MOI}}=112.11$ days, while HOI takes place $T_{\mathrm{HOI}}=173.25$ days after launch. 
Table 4: MOI problem. Optimal costs $\left(\mathrm{C}_{2} \mathrm{in} \mathrm{m} / \mathrm{s}\right)$ for ditferent launching velocity perturbations and dralays in first trajectory correction maneuver.

\begin{tabular}{|c|c|c|}
\hline$T C W 1_{\min }(\tan y s)$ & $e_{0}^{U}(\mathrm{~m} / \mathrm{s})$ & $C(\mathrm{~m} / \mathrm{s})$ \\
\hline \multirow[t]{5}{*}{3} & -3 & 45.1427 \\
\hline & 4 & 55.6387 \\
\hline & 5 & 65.9416 \\
\hline & -6 & 76.7144 \\
\hline & -7 & 87.3777 \\
\hline \multirow[t]{5}{*}{4} & -3 & 49.1817 \\
\hline & -4 & 61.5221 \\
\hline & -5 & 73.4862 \\
\hline & -6 & 85.7667 \\
\hline & -7 & 99.3405 \\
\hline \multirow[t]{4}{*}{5} & -3 & 53.9072 \\
\hline & -4 & 66.8668 \\
\hline & -5 & 81.1679 \\
\hline & $\begin{array}{l}-6 \\
-7\end{array}$ & $\begin{array}{r}94.3630 \\
109.2151\end{array}$ \\
\hline
\end{tabular}

Once we select a region of the stable manifold by selecting an appropriate initial guess trajectory, we can perform the same type of analysis as done for the HOI problem of $\S 3.1$. In what follows, we consider the case in which we correct for perturbations in launch velocity by seeking optimal MOI trajectories in Region B, that is, on the far side of the halo from the Earth. For given values of $\epsilon_{0}^{v}$ and $T C M 1_{\min }$, we first compute an MOI initial guess trajectory with $T_{0}=1.50$ and $T_{S}=0.75$ and then use the staggered optimization procedure described in $\S 3$ to find an optimal MOI trajectory in this vicinity.

We present results from such computations in Table 4. It can be seen that the optimal MOI trajectories are very close (in terms of their associated cost function $C_{2}$ ) to the corresponding HOI trajectories. These results can be understood if we recall that the nominal transfer trajectory that we use in our experiments actually inserts onto the halo orbit directly as opposed to the manifold. To take full advantage of the stable manifold in correcting for launching errors, one may need to start with a nominal transfer trajectories that insert onto the stable manifold. For missions that are designed to have such nominal transfer trajectories, correction trajectories that also insert onto the stable manifold are expected to be much more efficient than those obtained with the current formulation of the problem.

\section{Conclusions and Future Work}

This paper explores new approaches for automated parametric studies of optimal trajectory correction maneuvers for a halo orbit mission. Using the halo orbit insertion approach, for all the launch velocity errors and $T C M 1_{\min }$ considered we found optimal recovery trajectories. The cost functions (fuel consumption in terms of $\Delta V$ ) are within the allocated budget even in the worst case (largest $T C M 1_{\text {min }}$ and largest launch velocity error).

Using the stable manifold insertion approach, we obtained similar results to those found using HOI targeted trajectories. The failure of the MOI approach to reduce the $\Delta V$ significantly may be because the optimization procedure (even in the HOI targeted case) naturally finds trajectories 'near' the stable manifold. We will investigate this interesting effect in future work. 
The main contribution of dynamical systems theory to the problem of finding optimal recovery trajectories is in the construction of good initial guess trajectories in sensitive regions which allows the optimizer to home in on the solution. We feel that this aspect of our work will be important in many other future mission design problems. Many missions in the future will also require the use of optimal control in the context of low thrust. The software and methods of this paper can be used with little change for such problems. In fact, the techniques of this paper are applicable to a variety of problems and we plan to investigate them and related issues in future publications.

\section{References}

Ascher, U. M. and L. R. Petzold [1998] Computer Methods for Ordinary Differential Equations and Differential-Algebraic Equations, SIAM, 1998.

Ascher, U. M., R.M.M. Mattheij, and R. D. Russell, Numerical Solution of Boundary Value Problems for Ordinary Differential Equations, Society for Industrial and Applied Mathematics (SIAM) Publications, Philadelphia, PA, 1995. ISBN 0-89871-354-4.

Bertsekas, D.R. [1995] Nonlinear Programming, Athena Scientific, Belmont, Ma.

Bischof, C., A. Carle, G. Corliss, A. Griewank, and P. Hovland [1992], ADIFORgenerating derivative codes from Fortran programs, Scientific Programming, 1, 11-29.

Bischof, C., A. Carle, P. Hovland, P. Khademi, and A. Mauer [1998] ADIFOR 2.0 Users' Guide, MCS Division, Argonne National Laboratory, Technical Memorandum No. 192, June 1998.

Brenan, K. E., S. L. Campbell, and L. R. Petzold [1995] Numerical Solution of Initial-Value Problems in Differential-Algebraic Equations, SIAM Publications, Philadelphia, second ed.

Farquhar, R.W., D.P. Muhonen, C.R. Newman and H.S. Heuberger [1980] Trajectories and orbital maneuvers for the first libration-point satellite, J. Guidance and Control. 3, 549-554

Gill, P. E., L. O. Jay, M. W. Leonard, L. R. Petzold and V. Sharma [1998], An SQP Method for the Optimal Control of Large-Scale Dynamical Systems, submitted.

Gill, P. E., W. Murray, and M. A. Saunders [1997] SNOPT: An SQP algorithm for large-scale constrained optimization, Numerical Analysis Report 97-2, Department of Mathematics, University of California, San Diego, La Jolla, CA.

Gill, P. E., W. Murray, and M. A. Saunders [1998], User's Guide for SNOPT 5.3: A Fortran Package for Large-Scale Nonlinear Programming, Department of Mathematics, University of California, San Diego, La Jolla, CA. 
Gill, P. E., W. Murray, and M. H. Wright [1981], Practical Optimization, Academic: Press, London and New York.

Gómez, G., J. Masdemont and C. Simó [1993], Study of the transfer from the Earth to a halo orbit around the equilibrium point $L_{1}$, Cel. Mech. and Dyn. Astro. 56, 541-562 and 95, (1997), 117-134.

Howell, K. C., B. Barden and M. Lo [1997] Application of dynamical systems theory to trajectory design for a libration point mission, The Journal of the Astronautical Sciences 45(2), 161-178.

Howell, K. C. and H. J. Pernicka [1988] Numerical Determination of Lissajous Trajectories in the Restricted Three-Body Problem, Celestial Mechanics, 41, $107-124$.

Jorba, À. and J. Masdemont [1999] Dynamics in the center manifold of the collinear points of the restricted three body problem, Physica $D, 132,189-213$.

Koon, W.S., M.W. Lo, J.E. Marsden and S.D. Ross [2000] Heteroclinic connections between periodic orbits and resonance transitions in celestial mechanics, Chaos, to appear.

Li, S. and L.R. Petzold [1999], Design of New DASPK for Sensitivity Analysis, UCSB Department of Computer Science Technical Report.

Llibre, J., R. Martinez and C. Simó [1985] Transversality of the invariant manifolds associated to the Lyapunov family of periodic orbits near L2 in the restricted three-body problem, Journal of Differential Equations 58, 104-156.

Lo, M., B.G. Williams, W.E. Bollman, D. Han, Y. Hahn, J.L. Bell, E.A. Hirst, R.A. Corwin, P.E. Hong, K.C. Howell, B. Barden, and R. Wilson [1998] Genesis Mission Design, Paper No. AIAA 98-4468.

Maly, T. and L. R. Petzold [1996] Numerical methods and software for sensitivity analysis of differential-algebraic systems, Applied Numerical Mathematics 20, $57-79$.

Parker, T. S. and L. O. Chua [1989] Practical Numerical Algorithms for Chaotic Systems, Springer-Verlag, New York.

Petzold, L.R., J. B. Rosen, P. E. Gill, L. O. Jay and K. Park [1997] Numerical Optimal Control of Parabolic PDEs using DASOPT, Large Scale Optimization with Applications, Part II: Optimal Design and Control, Eds. L. Biegler, T. Coleman, A. Conn and F. Santosa, IMA Volumes in Mathematics and its Applications, 93, 271-300.

Serban, R. [1999] COOPT - Control and Optimization of Dynamic Systems - Users' Guide, Report UCSB-ME-99-1, UCSB Department of Mechanical and Environmental Engineering. 